\title{
J.R. Léveillé : habiter l'espace manitobain
}

Kathleen KELLETT, Université Ryerson

Le soleil du lac qui se couche ${ }^{1}$ de J.R. Léveillé est à la fois une célébration de l'espace manitobain et une exploration des rapports entre les êtres humains et les espaces qu'ils habitent. Alors que son roman précédent Une si simple passion tournait autour de Montréal, Paris et New York, Léveillé situe ce récit carrément au Manitoba. La ville de Winnipeg y paraît comme centre de vitalité artistique et cosmopolite. Les rues, les bâtiments et les monuments spécifiques de la ville intègrent l'espace de l'imaginaire alors qu'ils sont nommés, habitant ainsi le texte comme la ville : le Central Park, le coin de Portage et Main, l'Église du Saint-Rosaire, le centre Artspace, entre autres. Le nord du Manitoba, Cross Lake, Setting Lake (« Le lac qui se couche » éponyme), Wabowden, Thompson paraissent dans leur spécificité toponymique. Ainsi, le paysage des Prairies et l'espace urbain de Winnipeg et de la ville avoisinante Saint-Boniface se révèlent dans toute leur diversité ; de même, l'espace habité, l'architecture, l'espace public, ainsi que l'espace intime méritent une attention particulière ici. Il est à noter que Léveillé dédie le roman à l'architecte franco-manitobain Étienne Gaboury, à la communauté de Saint-Laurent dans le nord du Manitoba ainsi qu'à «tous [ses] amis du Manitoba ». Cette évocation de l'architecte renommé, de l'espace communautaire et du territoire convivial de la province souligne les liens étroits entre l'être humain et l'espace où l'on vit. Dans son article «L'évolution tranquille et la modernité culturelle », Léveillé a signalé l'importance de Gaboury au début des années 1960 à l'intérieur du renouveau culturel franco-manitobain, lui attribuant «une dynamique architecturale qui transforme le visage du vieux Saint-Boniface », au cœur même de la francophonie manitobaine (Sondes, 59). Sept ans après la parution du Soleil du lac qui se couche, Léveillé collabore avec Gaboury en créant un mince recueil de poésie intitulé L étang du soir, les poèmes d'Ueno Takami, y compris les Saisons intégrées. Léveillé et Gaboury ont donc « traduit de l'imaginaire » (page de titre) les poèmes du recueil de Ueno Takami, ce poète fictionnel du roman. S'il est vrai, d'après l'hypothèse de Simon Harel, que « toute forme narrative est récit d'espace, c'est-à-dire aménagement d'un lieu habité » (48; italiques dans l'original) ou oikos, ce roman de Léveillé offre surtout une interrogation sur les rapports entre les êtres humains, l'espace qu'ils habitent et l'espace qui les entoure. 
Dans Le soleil du lac qui se couche, la thématique de l'architecture, de la construction et de l'espace habité se manifeste tant sur le plan diégétique que sur le plan stylistique grâce à un foisonnement d'images littéraires. Les noms des architectes des Prairies dont Gaboury et Douglas Cardinal côtoient ceux des architectes internationaux tels qu'Antonio Gaudi, Frank Lloyd Wright et Tadao Ando. Ce sont des architectes dont s'inspire la narratrice, jeune Métisse $\mathrm{du}$ nom d'Angèle, en voie de réaliser sa vocation. Son amant Ueno Takami est poète et professeur de littérature, spécialiste d'Ezra Pound, poète américain fortement influencé par l'esthétique japonaise du haïku (Hakutani 69-70). Angèle songe à ses rencontres avec Ueno lors du séjour de celui-ci à Winnipeg où il veille à la mise en page de son recueil de poésie, L'étang $d u$ soir. Comme le remarque Benoît Doyon-Gosselin, la configuration spatiale principale du roman se manifeste comme une oscillation entre «deux pôles antinomiques » (Herméneutique, 271) : celui du sud, du centre, de la ville par opposition à celui du nord, de la périphérie, de la nature (272). Dans le contexte du motif architectural, d'autres oppositions s'imposent ici également, entre l'espace intérieur et extérieur, entre public et privé, entre profane et sacré. Finalement, grâce aux illustrations qui font partie intégrante de l'œuvre de Léveillé, il faut tenir compte de la représentation de l'espace au niveau paratextuel ainsi qu'au niveau diégétique.

\section{L'espace paratextuel}

Le soleil du lac qui se couche reprend souvent des correspondances entre l'architecture et d'autres formes d'expression artistique telle que la musique, la poésie et la peinture. La célébration des arts va de pair ici avec le métissage culturel au cœur du roman de Léveillé, thématique qui, selon Lise Gaboury-Diallo, en constitue « la pierre angulaire » (143). Ce thème se manifeste de plusieurs façons ; par exemple, Ueno Takami enseigne la poésie de Pound dont l'esthétique de l'imagisme reflète l'influence de la poésie japonaise. De la même façon, il présente à Angèle la musique de Jean-Pierre Rampal, fameux flûtiste français qui joue aussi de l'instrument japonais le shakuhachi. Au niveau du paratexte, le métissage culturel se manifeste notamment dans les six tableaux juxtaposés au texte de Léveillé, l'œuvre de l'artiste manitobaine Lorraine Pritchard qui s'inspire également de l'esthétique japonaise : «Some of my works integrate painting and collage with washi (handmade Japanese paper). They originate from the impulse to bring diverse elements together. The inherent theme is integration. Integration involves the relationships of one thing with another and the relationships of that part with the 
whole ». (1) Léveillé décrit l'œuvre de Pritchard comme « un havre de luminescence intérieure » (Sondes, 112). La couverture du roman présente l'image centrale du soleil au-dessus de la prairie, image tirée du tableau Émergence qui reparaît en entier vers la fin du roman, l'image de la couverture faisant partie d'un collage marqué d'une écriture griffonnée. Cette thématique d'une calligraphie en palimpseste paraît dans d'autres tableaux, tels Quad et Upper Signal. L'évocation de l'écriture japonaise à l'intérieur d'un art à prédominance nonfigurative souligne la puissance du langage comme moyen d'expression artistique. Nous sommes des êtres de langage - c'est le milieu où nous vivons. En contexte franco-manitobain, l'évocation de la prairie, habitat connu et rassurant, contraste avec l'illisibilité de cette écriture japonaise, effet de métissage culturel qui suscite un sentiment de dépaysement.

Le recueil de poèmes fictionnel d'Ueno Takami intitulé L'étang du soir accorde également une place importante au rapport entre texte et image : les gravures y évoquent, selon Angèle, «[...] l'étang dans la nuit et les poèmes des constellations dans le ciel. Un ciel renversé » (§ 85). En demandant à Angèle de traduire ses poèmes, Ueno souligne les correspondances entre l'écriture et l'architecture : «Tu t'intéresses aux arts, à la littérature, à l'architecture, et la traduction, c'est une espèce d'édifice » (§ 80). En faisant l'apprentissage de la traduction, Angèle approfondit sa compréhension de l'art en général : «C'est ce que j'ai fini par reconnaître : dès le premier soir, dans les décombres de mes petites constructions échouées, j'ai pris plaisir à tenter de traduire ces poèmes et à faire sortir ce que je ne croyais pas en moi » (§ 127). Dans le recueil L'étang du soir, il s'instaure un dialogue entre les poèmes de trois à cinq vers de Léveillé, et les dessins de Gaboury qui les accompagnent. Le poème en exergue du roman, «Les outardes / passent/ où les ouaouarons / demeurent », est repris dans ce recueil, une évocation des rapports entre habitant et habitat, entre nomade et sédentaire. De la même façon, dans le roman, en parlant d'un poème du recueil, Ueno s'identifie comme "oie » et désigne Angèle de «grenouille » (§ 81). La métaphore contraste celui qui s'envole et celle qui demeure. Le texte et l'image s'interpellent pour mettre en valeur l'entrecroisement des modes d'expression artistique. L'espace du recueil en est un de simplicité et de tranquillité, un portrait du paysage nordique où l'homme solitaire se retire du monde, un espace d'idylle qui contraste avec la diversité des lieux à l'intérieur du Soleil du lac qui se couche. 


\section{Winnipeg : espace du sud, espace du rêve}

Le lecteur entre dans le roman de Léveillé à travers les souvenirs d'Angèle dont on n'apprend jamais le nom de famille. Avec des résonances proustiennes, cette narratrice autodiégétique souligne la distance temporelle et intellectuelle par rapport à son jeune moi de vingt ans : «Il y a bien des années de cela. Je suis maintenant beaucoup plus vieillie, mais cette histoire tinte toujours en moi aussi clairement qu'une cloche dans le ciel vide » (§2). L'espace de l'énonciation est mal défini ici, mais on sait qu'Angèle a réalisé son ambition de faire des études en architecture au Manitoba. Le soleil du lac qui se couche est une histoire d'apprentissage ainsi qu'une histoire d'amour. Doyon-Gosselin le caractérise comme un « roman de la (dé)route »(Herméneutique, 215) : pour lui, le parcours d'amour d'Angèle et Ueno, qui implique de nombreux voyages entre Winnipeg et le nord du Manitoba, est une quête initiatique qui favorise l'épanouissement d'Angèle et l'aide à dépasser le sentiment de honte suscitée autrefois par son identité de Métisse au cœur de la société blanche.

Comme pour souligner son intégration à la communauté des artistes de sa ville, Angèle fait la rencontre initiale d'Ueno dans une galerie d'art lors d'une exposition de l'artiste Jack Crow Wing, fait qu'elle déplore puisqu'elle dit, «J'aimerais pouvoir dire que je l'ai rencontré à sa cabane dans le Nord du Manitoba » (§ 3). Grâce à Ueno, Angèle accède à une nouvelle perspective, une esthétique de l'hétérogène, qui implique non seulement une vision artistique mais une revalorisation de ses propres origines. Lorsqu'Ueno l'accueille en insistant positivement sur son identité métisse, elle se sent valorisée : «Car personne auparavant ne m’avait appelée Métisse sans provoquer chez moi un sentiment de honte » (§ 7). Attiré par sa nature double, il associe son aspect autochtone au rire et son aspect blanc à la mélancolie, sans juger ni l'un ni l'autre $(\S 5,6)$. Comme le souligne Gaboury-Diallo, cette mise en valeur de l'identité métisse va de pair dans ce roman avec la valorisation de l'hétérogène chez Ueno : «Pour le Japonais, le fait qu'Angèle soit Métisse, constitue un avantage pour elle, puisque 'sa nature hybride' reflète une sorte d'incarnation charnelle du yin et du yang » (148). Ueno l'encourage à poursuivre son rêve de devenir architecte. Effectivement, sa vision artistique aide Angèle à mieux formuler la sienne.

En songeant à cette carrière rêvée, la jeune Angèle se sent inspirée par les ouvriers autochtones qui excellent dans la construction des gratte-ciel grâce à leur agilité : «On disait que les Indiens d'Amérique n'avaient pas peur des hauteurs et possédaient un excellent équilibre. Je 
m'étais vue marchant allègrement sur une poutre d'acier dans le bleu du ciel. Il faut croire que l'idée que je me faisais de l'architecture était cette suspension du vide à mes pieds » (§ 7). L'architecture s'insère dans son espace personnel de façon assez ludique : lors de sa première visite au restaurant japonais Edohei sur la rue Ellice avec son amant, Angèle remarque les qualités architecturales du sushi : "J'y retourne chercher l'inspiration. Car je voyais dans le sushi des petites architectures modulaires. La base en riz modelé dans la main supporte une diversité de coupoles et de toitures » (§63). Pour meubler son appartement, elle s'achète un poisson rouge dans un bocal. Elle le nomme «Frankie » pour Frank Lloyd Wright tout comme elle a nommé son cactus « Tony » en l'honneur de l'architecte Antonio Gaudi (§ 57). L'espace intime d'Angèle est donc marqué de ses rêves et de ses ambitions personnelles. C'est à l'intérieur de cet espace aussi qu'elle garde, dans une boîte à cigare où elle met ses bijoux, le souvenir de son père, cadeau de sa mère à elle : «une griffe d'ours tenue en pendentif par une lanière de cuir » (\$10). Armée de ce talisman paternel, elle part se promener à Winnipeg à travers les lieux d'enfance.

L'association entre l'architecture et la culture autochtone sous-tend la vision artistique d'Angèle. Plus tard, en troisième année de ses études, lorsqu'elle rencontre le fameux architecte métis Cardinal, elle a l'impression qu'il la reconnaît comme Métisse car il répond à sa question en racontant une anecdote à propos du chef autochtone John Big Sky Carpenter, récit qui met en relief le sens inné des proportions chez cet homme. Cardinal constitue pour elle un modèle à émuler. Elle se sent inspirée, par exemple, par son église Sainte-Marie construite à Red Deer en Alberta, église qu'elle appelle Église Waskasoo en corrigeant l'erreur des Européens qui avaient confondu l'élan, appelé waskasoo en cri, avec le chevreuil roux (en anglais, « red deer »). Construite en 1968, cette église est un des chefs-d'œuvre de Cardinal, favorisant le curvilinéaire aux dépens du rectiligne conventionnel. Elle reflète la philosophie de l'architecture organique de Cardinal qui voit le cercle comme élément essentiel de la philosophie indigène laquelle met en relief l'épanouissement de l'individu par opposition à l'organisation hiérarchisante de la société occidentale (Cardinal, site officiel). Pour Angèle, le nom même de l'architecte, celui du bel oiseau rouge, lui convient sur le plan esthétique: "Cardinal, c'est un beau nom pour un architecte, et de surcroît pour un Indien » (§ 103). Métisse elle-même, Angèle admire la perspective de ce grand architecte franco-albertain métis qui fait fi des conventions architecturales pour créer des œuvres innovatrices parfaitement adaptées à leur environnement. 
Flâneuse, Angèle promène sur Winnipeg son regard de jeune architecte en devenir, sensible à ce milieu qu'elle habite depuis toujours. Elle nomme les rues de son enfance, celles de l'Exchange, de la paroisse Sacré-Cœur, les rues Furby, McDermot, Bannatyne. Sa patronne Mrs. Lydia, fleuriste, lui plaît non seulement à cause de son caractère doux et riant mais aussi parce qu'elle porte le nom d'une rue du quartier de son enfance, la rue Lydia. Bouleversée par son attraction naissante pour Ueno, sous le regard médusé de Mrs Lydia, Angèle se met à construire des arrangements de fleurs innovateurs mais pour la plupart peu susceptibles à intéresser les clients de l'hôpital. «On aurait dit que tu descendais d'une autre planète. As-tu suivi des cours d'ikebana ? », lui demande la patronne (§ 73, italiques dans l'original). Elle ne réussit à vendre qu'un bonsaï pour une convalescence de longue durée $(\$ 73)$. La créativité commence à suivre un chemin inattendu dans la mesure où les produits des fleuristes commerciaux, destinés à rassurer et non pas inspirer ni surprendre, ne conviennent pas à l'artiste. Dans la ville, Angèle recherche le réconfort de la verdure dans le Central Park, lieu d'enfance, qui ne correspond plus à ses souvenirs nostalgiques : «La belle clôture de fer forgé noir avait disparu. Le parc était plus ouvert, plus "nouveau”, moins intime qu'il m'avait semblé dans mon enfance [...] Les sentiers mieux délimités, mais moins avenants...moins sauvages...moins nature » (§ 14). La ville n’est donc pas entièrement coupée de la nature mais on pourrait parler d'une nature contaminée par l'influence de la ville, d'une nature plutôt apprivoisée et triste. Angèle se sent détachée de ce milieu d'enfance, se rendant compte que depuis quelques années, elle voit ce parc uniquement de son auto. Elle fait un rêve où la fontaine du parc jaillit sous le soleil et tout va pour le mieux. En réalité, Angèle est déçue de retrouver la vieille fontaine brisée mais Ueno la voit d'une autre manière et lui fait remarquer qu' «il y a une beauté dans l'incomplet » (§ 18). La beauté du paysage urbain valorisée par Ueno ne correspond pas aux attentes conventionnelles.

Le cercle d'amis artistes d'Angèle travaille à l'intérieur de l'espace cosmopolite de Winnipeg. Angèle visite le centre Artspace où son ami Aron Levi monte une installation. Dans sa représentation du paysage manitobain, Aron s'inspire de l'art autochtone nord-américain et australien ainsi que de l'art européen. S'agit-il de la reconnaissance du rôle primordial des peuples autochtones du territoire manitobain, leur oikos depuis des temps immémoriaux, ou bien de l'appropriation culturelle ? D'une part, l'art d'Aron est fidèle à l'esprit du métissage culturel prôné par Léveillé, une perspective qui tente de respecter toutes les traditions artistiques et culturelles ; d'autre part, ce jeune artiste emprunte des éléments artistiques à un peuple qui a subi 
la violence du colonisateur qu'Aron lui-même n'a jamais expérimentée ni n'expérimentera jamais. Il choisit de faire accompagner son installation de la musique aborigène du didjeridu: « un long hululement venant de l'autre bout du monde» qui, selon Angèle, apporte une dimension plus profonde à l'œuvre : «On aurait dit que les pièces avaient trouvé leur langue » (§ 134). Son installation suggère à Angèle l'œuvre du sculpteur Constantin Brancusi :

Les œuvres d'Aron ressemblaient à un vaste champ semé de poteaux totémiques. [...] Les totems étaient composés essentiellement de ces tiges de métal qu'on utilise dans le béton armé. Il y avait enfilé des flotteurs de bois. Il les avait tous ramassés sur les berges du grand lac Manitoba. «Là où ta famille fait la pêche », disait-il en me taquinant. [...] Il avait aussi taillé des branches ou de jeunes troncs qui arboraient une présence spiralée à la Brancusi. (§ 27)

Angèle remarque à quel point cet artiste roumain l'a influencée : «Brancusi est un sculpteur qui m'a donné d'extraordinaires idées d'habitation» (§ 27). C'est donc bien Brancusi adapté à l'espace manitobain. L'œuvre d'Aron plaît beaucoup à Ueno qui parle de «cette merveilleuse forêt rescapée des flots de la mortalité » (§ 134). Les barres d'armature et les flotteurs de bois évoquent la ville et la grande nature, réconciliées dans l'art.

Toujours dans cet espace urbain riche en projets artistiques, Angèle se promène dans les ateliers de Frank Rinella, l'imprimeur du même nom que celui qui a édité Le soleil du lac qui se couche ainsi que L'étang du soir de Léveillé et Gaboury. La jeune Angèle ne comprend pas toutes les implications sociales de l'architecture, toutes les controverses qui entourent par exemple la construction de l'Eglise du Saint-Rosaire où les habitants du quartier ont protesté avec succès contre une tentative de la paroisse de raser plusieurs maisons pour faire place à un terrain de stationnement pour l'église : «Je dois dire que lorsque je songeais à l'architecture, à l'époque, je ne pensais pas à toutes ces questions » (§ 58). Ce que l'architecte en herbe remarque surtout en ce qui concerne les bâtiments, c'est le rapport entre l'espace construit par les humains et la nature. Autrefois, selon la mère d'Angèle, le secteur où se trouve maintenant l'hôpital était « un grand parc boisé »: «Petit à petit, le béton a empiété sur la verdure » (§ 72). Pourtant, Angèle se plaît à son travail dans le Centre des Sciences de la Santé parce qu'elle aime l'introduction de la nature «à l'intérieur de cet édifice si clinique » (§ 72). Elle parle avec approbation de l'aile psychiatrique de ce bâtiment, «véritable bouffée d'air frais, pleine de lumière et de jardins intérieurs » (§72). Elle ne méprise pas toutefois les possibilités esthétiques du béton, par exemple, dans son appréciation de la ville de Winnipeg: «J'avais, comme je l'ai dit, l'impression de me promener dans une forêt de pierre et de ciment et de verre » (§58). Sa 
vision se rapproche de celle de Gaboury lui-même : «It is extremely important to remember that in architectural terms space has value and significance only in human terms. If a space does not provide joy, protection, inspiration to its users, it is not truly an architectural space. Space related to the human dimension is architecture » (247).

\section{Le Nord : l'espace de la quête}

C'est grâce à Ueno qu'Angèle découvre le nord du Manitoba. Il l'emmène avec lui à sa cabane près de Thompson. Au cours du trajet, elle est fascinée par la transformation graduelle de la route qui lui semble d'abord «longue et désolée », des arbres espacés où des incendies les ont réduits à « un spectacle de squelettes » mais, comme elle le dit, «Petit à petit le paysage du Nord prend de l'envergure, l'épinette noire, et la blanche s'imposent, du tremble et du bouleau se mêlent à du pin gris et du mélèze $[\ldots]$ »(§ 95). On remarque la spécificité du langage pour désigner les arbres, ce qui suggère une étroite familiarité avec la terre du nord, acquise par la narratrice autodiégétique au cours de cette histoire d'amour. Il s'agit d'une beauté tout autre que celle de Winnipeg, malgré la «clarté blanche » (§58) de la ville. En faisant allusion à la population importante de Métis dans cette région, Ueno lui dit, «C'est un peu ton pays » (§ 92). Angèle devrait pouvoir se sentir impliquée dans cet oikos mais elle en est aliénée. Elle ne peut pas entièrement s'identifier à ces gens du nord qui parlent le métchif, « français où se mêlait de l'ojibwa ou du cri, et parfois un peu d'anglais » (§ 92), alors qu'elle n'avait appris que ce qu'on appelle « le bon français » à son école de la paroisse Sacré-Cœur à Winnipeg. À l'intérieur de ce milieu francophone minoritaire, apprendre le français à Winnipeg relève pour elle du «miraculeux » (\$94), alors que pour la communauté autochtone, la langue française, imposée par la colonisation, est plutôt une force destructrice : «J'ai appris qu'ils ont longtemps eu honte de parler leur langue, qu'ils ont dû lutter contre l'éducation 'française' qu'ils recevaient dans les congrégations. J'aimais leur façon d'assouplir les mots et les expressions et de chantonner les phrases » (§ 92). Comme eux, elle a connu un sentiment de honte envers ses racines autochtones. À l'école, comme elle ne ressemblait pas physiquement à sa sœur qui n'avait pas le même père, on croyait qu'elle avait été adoptée. Pourtant, sa sœur dit en riant « Nous sommes des jumelles » (§ 64). L’amour familial fournit un rempart de solidarité contre les préjugés sociaux.

Le soleil du lac qui se couche fait valoir une architecture qui relie l'être humain à la nature qui l'entoure. Angèle décrit ainsi la cabane que le poète japonais a construite avec l'aide 
de la communauté métisse de la réserve de Cross Lake qui offre à l'étranger l'hospitalité des lieux. Or, comme l'indique Harel, « L'hospitalité liée à l'oikos configure l'espace social » (115). La cabane d'Ueno offre un exemple parfait d'une esthétique dérivée du métissage culturel :

C'était une cabane qui ressemblait à la fois à un tipi, à une cabane en rondins typiques, et à l'architecture japonaise moderne. Elle était simple et stylisée. C'est l'intégration des proportions et le jeu des matériaux qui faisaient qu'elle ne jurait pas en ce lieu. Mais elle nous obligeait à réfléchir sur toute la question de l'habitat dérivé de son origine dans la nature. $(\S 101)$

La pluie qui tombait sur le bois et le fer du toit donnait une musique tranquillisante ; les couleurs cadraient bien avec « le paysage d'arbres et de roc » (§ 101). Comme le dit Doyon-Gosselin, « Il s'agit du parfait équilibre entre le passé japonais de Ueno, le caractère nord-américain des rondins et l'influence des traditions amérindiennes locales » («Autant», 129). Ueno compare la cabane «aux maisons de son pays natal, à la maison de ses parents plus particulièrement » (§ 89). La tranquillité de la nature va de pair ici avec le réconfort de la maison d'enfance. Cette construction illustre le principe bouddhiste $z^{2}{ }^{2} \mathrm{du}$ «wabi-sabi », qu'Ueno définit ainsi : «Les choses wabi-sabi sont le registre tangible du passage et de l'effet de l'air, du vent, du soleil. La rouille, la décoloration, la déformation, les fissures en sont les caractéristiques essentielles » (§ 27). Pour sa part, Angèle parle de «l'aménagement paysager qu’il avait réussi sans détonner en plein cœur du grand Nord» (§ 102). On pense à l'architecture curvilinéaire de l'église SaintMarie réalisée par Cardinal. De même, Gaboury préconise une architecture régionale qui tiendrait compte du climat et de la géographie des Prairies : «To me, the natural elements are the most powerful source of design inspiration. We have somehow forgotten that the sun rises in the East and sets in the West and that this rhythm establishes the cadence of life; that our activities are programmed by the pulse of night and day » (246).

L'espace intérieur de la cabane reflète le penchant syncrétiste d'Ueno pour la juxtaposition insolite des objets de haute culture et de culture populaire, ramassés au cours de sa vie. DoyonGosselin a déjà remarqué le caractère hétérogène des objets à l'intérieur de la cabane et du camion d'Ueno (Herméneutique, 281). Angèle s'étonne devant l'amas d'objets d'art chez Ueno :

Des couvertures indiennes bien sûr, des tapis sud-américains, des masques mexicains, de la poterie japonaise, un crâne de taureau à longue cornes était accroché à un mur; sur un autre, une peinture abstraite - une très petite toile de Jackson Pollock, je n'arrivais pas à en croire mes yeux, c'était un original; un labyrinthe de noir et de blanc s'enfonçant dans le marron. (§ 109) 
Le tableau rappelle le commentaire d'Ueno à propos de l'art de la gravure : «Le noir, disait Ueno, est au calligraphe japonais ce que la neige est à l'Inuk. Le blanc est un univers, le noir aussi »(§ 82). S'il est difficile de passer sous silence l'opposition ici entre l'artiste japonais associé à la culture et l'Inuk associé plutôt à la nature, il faut aussi noter que, conformément à l'art poétique d'Ueno, ici, à travers l'expression artistique, les univers culturels autochtones, asiatiques et euro-américains se rejoignent.

En sondant le rapport entre habitat et habitant, il faut aussi s'interroger sur la signification de l'autre cabane qu'Ueno a fait construire sur sa propriété du nord, loin des agréments urbains. Caché dans les bois, au bout d'un petit sentier, dans « un enclos déboisé, presque circulaire », Angèle remarque un autre édifice muni d'électricité, où se trouve un générateur et une antenne parabolique, une salle de douche, la lessive, l'ordinateur, le téléphone et le téléviseur : «Un véritable centre de télécommunication » (\$ 113). Comme le remarque Angèle, Ueno préfère agir «comme si ces éléments de la vie moderne n’existaient pas » (§ 113). Il explique ainsi la fonction de cette deuxième cabane : «Pour me tenir au courant et mener certaines affaires que j'ai en Orient » (\$ 113). Pourtant, on pourrait aussi y voir une tentative de cacher le malaise que provoque la technologie, perçue comme une forme de contamination de la nature et de l'espace intime : «Alors j'ai senti en venant ici que si je voulais vivre comme je l'entendais, je devais séparer l'espace que j'habitais de celui où j'étais appelé à maintenir mon contact avec le monde extérieur » (§ 114). Ueno compare ses deux cabanes à celles de Grey Owl, personnage historique très équivoque dans l'imaginaire canadien : «C'est comme les deux cabanes de Grey Owl, m'at-il raconté. Tu as entendu parler de ce Britannique qui s'est fait passer pour un Indien ?» (§ 114). Grey Owl était un écrivain et un conférencier très populaire dans les années 1930, connu pour ses récits à propos de la vie autochtone et de la nature canadienne, démasqué après sa mort en 1938 comme un immigrant britannique du nom d'Archibald Stansfeld Belaney (Dawson 390). Comme l'explique Ueno, en travaillant pour le Service des parcs dans le Nord de la Saskatchewan, Grey Owl avait fait construire une cabane en rondins au bord du lac Ajawaan, logis qu'il partageait avec sa femme Anahero, d'origine Mohawk, ainsi qu'avec des castors qui pouvaient pénétrer dans la cabane à travers l'eau du lac. Lors de la naissance de son enfant, Grey Owl a fait construire une autre cabane tout près pour y habiter avec sa femme et leur fille. Il faut dire que Grey Owl est mieux connu de nos jours pour son usurpation de l'identité autochtone que pour ses récits au sujet des grands espaces canadiens. Sa présence dans le texte de Léveillé 
souligne le danger de glissement, l'équivoque potentiel entre le métissage culturel et l'appropriation culturelle. Dans «The 'I' in Beaver: Sympathetic Identification and SelfRepresentation in Grey Owl's Pilgrims of the Wild», Carrie Dawson note que, malgré la force de l'écriture de Belaney qui prêchait la préservation des grands espaces, cet homme paraît comme un être isolé de toute communauté humaine (397). Selon Dawson, la vision que Grey Owl évoque de la grande nature et de la vie des autochtones promeut le stéréotype d'un mode de vie et d'un peuple en voie de disparition, perspective qui n'est pas destinée à favoriser l'épanouissement des communautés indigènes (400). Par contre, en construisant sa demeure, Ueno collabore avec la communauté du nord à forte population métisse. Toujours est-il qu'en créant une cabane pour cacher les appareils de la technologie moderne dont il dépend pour son travail, le personnage véhicule une notion de l'incompatibilité entre la grande nature du Nord et la vie moderne soutenue par la technologie qui risque de contaminer les grands espaces sauvages.

En analysant le trajet entre Winnipeg et le nord du Manitoba, Doyon-Gosselin met en relief l'importance de l'apprentissage dans ce roman : « Initiatique, ce déplacement dans l'espace plus symbolique que physique permet à la protagoniste de mieux se définir par rapport au monde où elle vit »(Herméneutique, 270). Citadine de Winnipeg, Angèle ne connaît pas le nord du Manitoba avant d'accompagner Ueno en route à sa cabane. Leur amour se développe au cours d'une série de visites, de trajets entre Winnipeg et la cabane près de Wabowden. En se promenant au bord du lac et à l'intérieur de la forêt qui forment la propriété de Takami, les deux amants entendent «un bel et étrange hululement» (§ 148) qu’Ueno identifie comme la chouette cendrée, symbole de Minerve, déesse grecque de la sagesse. Doyon-Gosselin y voit le symbole de la maturation d'Angèle au cours de cette quête (Herméneutique, 287). Peu après, deux annonces bousculent cette histoire d'amour : celle de la mort imminente d'Ueno ( $\$ 150)$ et celle de la naissance anticipée de leur enfant ( $\$ 158)$.

\section{De l'espace profane à l'espace sacré : l'aboutissement d'une quête}

Le récit initiatique aboutit à l'établissement d'un rapprochement entre l'espace profane et l'espace sacré associé à l'amour d'Angèle et d'Ueno. La conception de l'enfant d'Angèle et du vieux poète, enfant qu'Ueno propose d'appeler « Isaake Takami », relève d'une spiritualité issue en partie des grands récits judéo-chrétiens. Déjà le nom d'Angèle relève du sacré ; celui de son 
fils anticipé aussi. Le nom «Isaac » est lourd de signification dans le contexte judéo-chrétien : c'est le fils d'Abraham et de Sarah, fils que Sarah a eu tard dans sa vie après des années d'attente. Il sera l'héritier d'Abraham. Âgé de soixante-quatre ans et atteint d'une maladie mortelle mystérieuse, Ueno a bien assuré Angèle qu'il était peu probable qu'il puisse devenir père ; il lui dit : «Oh ! Tu sais, à mon âge, avec ma condition physique, je ne crois pas qu'il y ait suffisamment de mouches à feu pour franchir le barrage des bouddhas. Ce serait là, non pas un miracle, mais un agrément insoupçonné et incontournable de l'univers » (§ 159). Pour GabouryDiallo, cette naissance est la réalisation de l'idéal du métissage culturel chez Léveillé : «Le métissage se perpétue et, avec la naissance de l'enfant, la continuité et le consentement nécessaire au dialogue sont assurés » (151).

Plusieurs références relient la philosophie d'Ueno et sa recherche du sacré à la tradition judéo-chrétienne : en parlant de Setting Lake, dont le nom algonquin veut dire « là où l'on place les filets », il en caractérise la pêche comme «Miraculeuse » (§ 101). On pense au Christ qui disait aux disciples où placer les filets pour avoir la meilleure pêche (Jean 21:6). Dans sa cabane, son petit jardin zen, plutôt que de consister en du sable ratissé, se compose des noyaux de toutes les olives qu'il a mangées au cours des années; il l'appelle ludiquement son «jardin des oliviers » ( $(108)$. Ueno retrouve le sacré lui-même dans la sexualité féminine, le principe créateur féminin, ce qui est souligné par la mention de la reproduction d'un tableau du peintre Gustave Courbet parmi les biens de l'artiste dans la cabane du Nord. Angèle en parle : «Il y avait même une petite toile qui au début me semblait plutôt dévergondée, que je suis arrivée à connaître au cours des mois qui ont suivi. C'était une reproduction de L'origine du monde de Courbet. - C'est là que je prie, a-t-il dit » (§ 144). Ce tableau très controversé représente une femme couverte d'un drap qui laisse voir seulement un sein et, en gros plan, le sexe de la femme. La première réaction d'Angèle n'est pas surprenante; depuis sa création, cette œuvre, commanditée par le diplomate turc Khalil-Bey, a souvent été considérée comme pornographique (Desbuissons 16). Par contre, le titre même du tableau constitue une affirmation du sexe féminin en tant que l'origine de la création et lieu de procréation. Pour souligner les correspondances transculturelles, Léveillé fait suivre ce passage d'un autre où Ueno cite le poète japonais ancien Ikkyu. Il s'agit d'un poème intitulé « Le sexe de la femme », qui parle de « la bouche originelle » qui est aussi « le lieu de naissance de tous les Bouddhas des dix mille mondes » (§ 145). Étant donné la controverse qui entoure le tableau de Courbet qui, en ne représentant que le sexe de la 
femme, associe la créativité féminine exclusivement à la sexualité et à la fonction biologique de procréation, c'est-à-dire, à la nature plutôt qu'à la culture, ces références sont quelque peu équivoques. Il s'agit avant tout d'une célébration de la sexualité créatrice et cela, d'une perspective résolument masculine.

Dans la représentation de cette naissance miraculeuse d'un fils, on pourrait voir un fantasme strictement masculin, le désir de perpétuer sa lignée, d'avoir un fils qui continue l'œuvre du père (Kellett-Betsos 164). Par contre, le mot «Isaac » veut dire en hébreu « celui qui rit ». C'est donc un excellent présage pour l'avenir de l'humanité. Dès leur première rencontre, Ueno associe le rire au côté métis d'Angèle et la mélancolie à son côté blanc. Angèle remarque : « Tu as choisi le titre, L'étang du soir, parce que tu penses que ce sera ton dernier livre. C'est pourquoi j’y sens une couche d'amertume, de tristesse » (§ 151). Ce à quoi Ueno répond : «La tristesse est la condition ineffable de l'univers. Pourtant nous sommes appelés à être heureux » (§ 151). Ueno privilégie le principe de la création dans la joie, qu’il s'agisse de création artistique ou de procréation. Dans le recueil de poèmes non-paginés de Léveillé et de Gaboury, le jeu de mots sur «l'étang de la pleine nuit» du premier poème et «pleine nuit de l'étant» du dernier suggère que l'étang d'Ueno n'est pas un espace référentiel décrit sur le mode réaliste, mais surtout l'espace de l'existence, l'habitat de l'esprit. Comme la cabane principale d'Ueno, ce lieu est surtout solitaire et dépourvu de technologie moderne; le poète se plaint : «que de routes / pour me retrouver / seul ». Dans le roman, en se penchant sur son jeune moi, la narratrice autodiégétique se rend compte de l'importance de la joie de la création artistique non seulement pour sa compréhension de l'espace architectural mais aussi pour l'espace humain du Manitoba en tant qu'oikos, pour ses artistes, ses cultures diverses et sa propre identité métisse. C'est à travers l'art et l'étude de l'architecture qu'Angèle renoue avec la communauté indigène, grâce non seulement à Ueno mais aussi à l'influence de Cardinal, de ses créations artistiques et de son histoire de John Big Sky Carpenter. La richesse innovatrice de l'architecture adaptée aux Prairies, représentée notamment par Cardinal et Gaboury, assume sa place à l'intérieur de l'expression artistique du monde entier, sans perdre de sa spécificité rattachée à un habitat particulier.

Tout en célébrant la beauté du paysage nordique ainsi que celle de l'espace urbain, $L e$ soleil du lac qui se couche a tendance à présenter la technologie et le béton comme des éléments de contamination qui empiètent sur la nature. On décèle aussi une certaine ambiguïté par rapport 
à la femme qui semble s'associer de près à la nature plutôt qu'à la culture ; c'est notamment le cas de la vénération par Ueno du tableau de Courbet. Pourtant, le portrait d'Angèle que dessine Léveillé est celui d'une femme autonome, créatrice et intelligente, véritable Minerve selon Doyon-Gosselin ; c'est une architecte consciente de la complexité des rapports entre l'espace architectural et l'auto-épanouissement humain. Le roman de Léveillé nous invite à célébrer le métissage culturel chez des artistes tels que la peintre Lorraine Pritchard, le poète Ezra Pound et le flûtiste et joueur du shakuhachi Jean-Pierre Rampal, mais l'exemple de Grey Owl suggère aussi l'importance d'un regard critique qui tient compte de la tentation d'une appropriation culturelle peu respectueuse des cultures de l'Autre. Par contre, l'espace évoqué par Léveillé ici est celui d'une collaboration fructueuse entre des représentants de cultures diverses tels que l'architecte métis Douglas Cardinal, le poète japonais Ueno Takami, l'architecte métisse Angèle, l'artiste juif Aron Levi, le typographe italien Frank Rinella et toute la communauté métisse du nord du Manitoba. Ici, le métissage culturel s'associe étroitement à l'oikos dans la mesure où sa véritable richesse dépend de la valeur accordée à cette hospitalité, cette ouverture culturelle, mais seulement dans un contexte qui implique l'égalité, le respect mutuel, la convivialité et l'esprit de partage.

\section{Bibliographie}

Cardinal, Douglas. «Douglas Cardinal, architecte». http://www.djcarchitect.com/. Voir «Work: Saint-Mary's», «Philosophy: World View Presentation». Consulté le 7 novembre 2016.

Dawson, Carrie. "The "I" in Beaver: Sympathetic Identification and Self-Representation in Grey Owl's Pilgrims of the Wild». Greening the Maple ; Canadian Ecocriticism in Context. Dir. Ella Soper et Nicholas Bradley. Calgary : U of Calgary P, 2013. 387-405.

Desbuissons, Frédérique. «Voir le mal en face : L'origine du monde de Gustave Courbet». Ligeia, 1 octobre $1996: 14-22$.

Doyon-Gosselin, Benoît. « Autant en emportent les vents : portrait de la Métisse dans Le soleil du lac qui se couche de J.R. Léveillé ». J.R. Léveillé par les autres. Dir. Lise GabouryDiallo, Rosmarin Heidenreich et Jean Valenti. Saint-Boniface : Blé, 2005. 125-135.

---. Pour une herméneutique de l'espace ; l'œuvre romanesque de J.R. Léveillé et France Daigle. Québec : Nota bene, 2012. 
Gaboury, Étienne. « Towards a Prairie Architecture ». Prairie Forum 5.2 (1980) : 237-247.

Gaboury-Diallo, Lise. "Manifestation du "transculturel” et de "métissage” chez Ronald Lavallée et J.R. Léveillé, deux écrivains contemporains du Manitoba français ». J.R. Léveillé par les autres. Dir. Lise Gaboury-Diallo, Rosmarin Heidenreich et Jean Valenti. SaintBoniface : Blé, 2005. 137-156.

Gontrand, Marc. «Préface ». J.R. Léveillé. Le soleil du lac qui se couche. Montréal : BCF ; Saint-Boniface : Blé, 2009. 5-12.

Hakutani, Yoshinobu. «Ezra Pound, Imagism, and Japanese Poetics ». Haiku and Modernist Poetics. New York : Palgrave Macmillan, 2009. 69-88.

Harel, Simon. Les passages obligés de l'écriture migrante. Montreal : XYZ, 2005.

Kellett-Betsos, Kathleen. "Le soleil du lac qui se couche / The Setting Lake Sun ». J.R. Léveillé par les autres. Dir. Lise Gaboury-Diallo, Rosmarin Heidenreich et Jean Valenti. SaintBoniface : Blé, 2005. 157-165.

Léveillé, J.R. et Étienne Gaboury. L'étang du soir, les poèmes d'Ueno Takami. Saint-Boniface : Blé, 2008.

Léveillé, J.R. Le soleil du lac qui se couche. Ill. Lorraine Pritchard. Saint-Boniface : blé, 2001 ; Montréal / Saint-Boniface : BCF / Blé, deuxième édition, 2009.

---. Sondes ; essais et entrevues. Saint-Boniface : Blé, 2014.

---. Une si simple passion. Saint-Boniface : Blé, 1997.

Pritchard, Lorraine. « Lorraine Pritchard ». Women Artists in Canada, Collections Canada, 1996. http://www.collectionscanada.gc.ca/eppparchive/100/205/301/ic/cdc/waic/loprit/loprit_s.htm. Consulté le 7 novembre 2016.

\footnotetext{
NOTES

${ }^{1}$ Toutes les citations proviennent de la version originale et illustrée du Soleil du lac qui se couche de J.R. Léveillé, publiée en 2001 chez Les Éditions du blé.

${ }_{2}^{2}$ Pour une brève analyse de l'influence de la philosophie bouddhiste zen dans ce roman de Léveillé, voir la préface de Marc Gontrand écrite pour l'édition du Soleil du lac qui se couche publiée en 2009.
} 